\title{
Engaging Hospitalists in Antimicrobial Stewardship: The CDC Perspective
}

Arjun Srinivasan, MD

Division of Healthcare Quality Promotion, Centers for Disease Control and Prevention, Atlanta, Georgia.

Disclosure: Dr. Srinivasan has not received any funding for his contribution to this article.

The findings and conclusions in this report are those of the author and do not necessarily represent the views of the Centers for Disease Control and Prevention.

There has never been a more important time to promote efforts to improve the use of antibiotics in hospitals. The growing challenges of $C$. difficile infections and antimicrobial resistance can both be addressed, at least in part, through so called "antimicrobial stewardship" efforts. Data suggest that when the right antibiotics are prescribed at the right dose for the right durations patient outcomes are improved and healthcare dollars are saved. That improving antibiotic use is a win-win-win is not in question; what remains to be determined is how best to ensure that these efforts are implemented in all hospitals. I believe that hospitalists must play an important role in advancing antimicrobial stewardship. Journal of Hospital Medicine 2011;6:S31-S33.

\section{KEYWORDS: antimicrobial stewardship, Clostridium difficile, length of stay, quality improvement.}

Additional Supporting Information may be found in the online version of this article.

What if there was a quality improvement initiative that had been proven in multiple, peer-reviewed publications to improve individual patient outcomes, reduce the overall burden of antimicrobial resistance, and save healthcare dollars? Surely such an initiative would enjoy widespread, if not uniform, adoption by health care facilities. Antimicrobial stewardship is just such an intervention. Ensuring that hospitalized patients receive the right antimicrobial, at the right dose, at the right time, and for the right duration has been shown to reduce mortality, ${ }^{1}$ reduce the risks of Clostridium difficileassociated diarrhea, ${ }^{2}$ shorten length of stay, ${ }^{3}$ reduce overall antimicrobial resistance within the facility, ${ }^{4}$ and save money. ${ }^{5}$ Yet despite these benefits, antimicrobial stewardship programs and interventions are far from the norm in US hospitals.

There are 2 important myths about antimicrobial stewardship that likely contribute substantially to the gap between the recognized benefits and implementation of stewardship interventions. Dispelling these myths is a crucial step in promoting wider adoption efforts to improve antimicrobial use. The first myth stems from the very name "antimicrobial stewardship program," which has created a misperception that optimal inpatient antimicrobial use is only possible in settings with formal stewardship programs that are staffed by infectious diseases (ID) physicians and pharmacist. The best guidelines on implementing stewardship programs, developed by the Infectious Diseases Society of America and the Society for Healthcare Epidemiology of America, ${ }^{6}$ may have contributed to this misperception by suggesting that optimal programs require dedicated time from both an ID physician and an ID-trained pharmacist. However, many hospitals do not have ID physicians on staff, and the vast majority do not have access to an ID pharmacist who is comfortable with antimicrobial stewardship. Although these traditionally staffed programs have well-proven benefits and are an excellent goal, they are not feasible in many hospitals. However, different types of stewardship interventions, led by a variety of health care providers and specialists, also have well-proven benefits. Although these latter experiences are much less likely to appear in peer-reviewed medical journals, experts in antimicrobial stewardship indicate that they often hear about very successful stewardship interventions being led by groups like general clinical pharmacists, intensivists, and hospitalists. Workshops on antimicrobial stewardship are often full of attendees who are successfully improving antimicrobial use in facilities that represent the full spectrum of US hospitals: large and small, urban and rural, teaching and nonteaching. Indeed, I prefer the term "antimicrobial stewardship programs and interventions" to convey that improving antimicrobial use can be done, and done well, even without the ideally staffed program.

The second myth is that the only goal of stewardship programs and interventions is to stop clinicians from using antimicrobials. This misperception has led to counterproductive attitudes toward stewardship programs and interventions in some facilities. Without question, stopping unnecessary antimicrobial use is an important aspect of stewardship interventions that has well-established benefits for patients and hospitals. That one third to one half of all inpatient antimicrobial use might be unnecessary, combined with the growing problem of C. difficile, certainly supports the goal of reducing inappropriate antimicrobial use. However, the primary goal of stewardship is to optimize antimicrobial therapy. In many instances, this does involve stopping unneeded antimicrobials, and because stopping antibiotics 
has the most readily demonstrable benefits on patient and financial outcomes, interventions with this aim are the subject of nearly all published studies. However, anyone who has worked on stewardship interventions can describe numerous instances when the recommendation provided was to broaden or lengthen antimicrobial therapy. Moreover, surveys indicate that, far from viewing stewardship as an intrusion or infringement on their autonomy, clinicians appreciate and even want the assistance that these efforts provide. ${ }^{7}$

If stewardship has substantial proven benefits, can be implemented in nearly any hospital setting, and is welcomed by providers, what can be done to move toward broader implementation? I believe that engaging hospitalists more fully in stewardship efforts will be a critical step in this direction. Hospitalists already provide a substantial portion of all inpatient care in the United States, and the numbers of hospitalists are growing rapidly. Moreover, they are increasingly taking the lead in a variety of quality improvement initiatives. Hence, hospitalists are ideally positioned and well suited to move stewardship efforts forward. Some, including hospitalists, have also suggested that developing a practical "stewardship implementation framework" would be helpful in promoting these interventions.

This suggestion has led the Centers for Disease Control and Prevention's (CDC) Get Smart for Healthcare campaign to partner with the Institute for Healthcare Improvement (IHI) and a variety of external experts (including a hospitalist) to develop such a framework using the IHI's Driver Diagram and Change Package methodology. The driver diagram seeks to identify a core set of highly influential practices that lead to a desired outcome. For optimizing antimicrobial use, the primary drivers that were identified by experts include: 1) timely and appropriate initiation of antibiotics; 2) appropriate administration and de-escalation of therapy; 3) data monitoring and transparency (measuring and feeding back to clinicians data on antimicrobial use and resistance); and 4) improving stewardship infrastructure, knowledge, and engagement in antimicrobial stewardship efforts. Once these drivers were identified, the expert panel then identified a number of specific practices, or "change concepts," that would support progress toward each driver. Now that the Driver Diagram and Change Package has been drafted, the CDC and IHI are collaborating on a pilot testing effort and are working to ensure that a substantial number of the pilot projects are led by hospitalists. Our goal is that the Driver Diagram and Change Package will be honed and refined with the help of hospitalists so that the end result will be a highly implementable set of antimicrobial stewardship interventions that can be widely applied by hospitalists around the country.

However, we need not wait for finalization of the Driver Diagram and Change Package to begin a productive collaboration on antimicrobial stewardship. In addition to the project with IHI, Get Smart for Healthcare is working to identify a variety of resources that would be useful in implementing and improving stewardship efforts. To that end, we would love to hear from any hospitalists who would like to share their experiences with stewardship interventions or who have tools (eg, order sets), ideas (eg, particularly successful intervention projects), or success stories. They can be e-mailed to beu8@cdc.gov.

For now, I would like to suggest that there are 4 antibiotic quality improvement projects hospitalists would be ideally suited to lead. The first is ensuring that all antibiotic orders include a "Dose, Duration, Indication." Efforts to improve antibiotic use are often hampered because the nonprescribing providers are not sure why the patient is on antibiotics. This problem is amplified when patients are transitioned from one provider to another or when multiple providers are involved. Specifying the duration and indication in all antibiotic orders will ensure that treatments continue for the right amount of time and would allow therapy to be stopped if the initially suspected infection is ruled out or altered if another infection is identified. The second improvement project is developing a process to ensure that any patient with a positive blood culture is on the appropriate therapy. This is a relatively straightforward intervention that is based on the patient's own microbiology results, and it ensures the optimal therapy of a serious infection. Third is the development of an intervention to encourage the reassessment of patients who are started on antibiotics for communityacquired pneumonia (CAP). Several hospitalists have suggested that the pressure to initiate therapy quickly in cases of CAP often leads to overtreatment. Interventions that encourage a reexamination of the CAP diagnosis when the clinical situation has stabilized would likely reduce this overtreatment. And the fourth improvement project is ensuring that urinary tract infections (UTIs) in hospitalized patients are properly diagnosed and treated. Work done by hospitalists at the University of Michigan suggests that improving the diagnosis and treatment of UTIs would have a significant impact on improving antibiotic use. ${ }^{8}$ Currently, the CDC is collaborating with these investigators to develop protocols and tools to improve the treatment of inpatient UTIs.

The time to promote aggressive implementation of antimicrobial stewardship interventions has come. Clinicians are increasingly encountering infections for which there are very limited or, in some cases, no good treatment optionsand there are very few new antibiotics on the horizon. Many groups are advocating for expanded efforts to develop new antibiotics. ${ }^{9}$ Although this is crucial, it is just as important that we work now to aggressively improve the use of the agents we have. Not only might this extend the life of our current agents, but it will also help ensure that any new agents will enjoy longer periods of effectiveness. Indeed, failing to inextricably link the development of new antibiotics with efforts to improve antibiotic use is akin to buying a new car to drive on a road full of potholes. Fortunately, there are a number of interventions that have proven successful; we now need to determine how best to apply these interventions in more settings. We want and need the involvement of hospitalists in these efforts. Yes, improving 
antimicrobial stewardship will require investments, but past experience tells us that the alternative could prove far more costly.

\section{Address for correspondence and reprint requests:}

Arjun Srinivasan, MD, Division of Healthcare Quality Promotion, Centers for Disease Control and Prevention, 1600 Clifton Rd, MS A35, Atlanta, Georgia 30333; Telephone: 404-639-2303; Fax: 404639-2647; E-mail: Beu8@cdc.gov. Received 29 July 2010; revision received 21 September 2010; accepted 22 September 2010.

\section{References}

1. Kollef MH. Inadequate antimicrobial treatment: an important determinant of outcome for hospitalized patients. Clin Infect Dis. 2000;31:S131-S138.

2. Valiquette L, Cossette B, Garant MP, et al. Impact of a reduction in the use of high-risk antibiotics on the course of an epidemic of Clostridium difficile-associated disease caused by the hypervirulent NAP1/027 strain. Clin Infect Dis. 2007;45(Suppl 2):S112-S121.

3. Omidvari K, De Boisblanc BP, Karam G, et al. Early transition to oral antibiotic therapy for community-acquired pneumonia: duration of therapy, clinical outcomes, and cost analysis. Respir Med. 1998;92:1032-1039.
4. Bantar C, Sartori B, Vesco E, et al. A hospitalwide intervention program to optimize the quality of antibiotic use: impact on prescribing practice, antibiotic consumption, cost savings, and bacterial resistance. Clin Infect Dis. 2003;37:180-186.

5. Agwu AL, Lee CK, Jain SK, et al. A World Wide Web-based antimicrobial stewardship program improves efficiency, communication, and user satisfaction and reduces cost in a tertiary care pediatric medical center. Clin Infect Dis. 2008;47:747-753.

6. Dellit TH, Owens RC, McGowan JE Jr, et al. Infectious Diseases Society of America and the Society for Healthcare Epidemiology of America guidelines for developing an institutional program to enhance antimicrobial stewardship. Clin Infect Dis. 2007;44:159-177.

7. Srinivasan A, Song X, Richards A, et al. A knowledge, attitudes and beliefs survey of housestaff physicians from various specialties concerning antimicrobial use and resistance. Arch Intern Med. 2004;164: 1451-1456.

8. Gandhi T, Flanders SA, Markovitz E, et al. Importance of urinary tract infection to antibiotic use among hospitalized patients. Infect Control Hosp Epidemiol. 2009;30:193-195.

9. Infectious Diseases Society of America. The $10 \times 20$ Initiative: pursuing a global commitment to develop 10 new antibacterial drugs by 2020. Clin Infect Dis. 2010;50:1081-1083. 